\title{
Questions de genre et développement durable: le potentiel de l'agroécologie dans le Nordeste du Pará, Brésil
}

\author{
Francesca Alice Centrone ${ }^{1,2, *}$, Jean-Philippe Tonneau ${ }^{3,5}$, Marc Piraux ${ }^{3,5,6}$, Nathalie Cialdella ${ }^{4,5}$, \\ Tania De Sousa Leite ${ }^{6}$, Angela Mosso ${ }^{1}$ et Angela Calvo ${ }^{1,2}$ \\ ${ }^{1}$ Département des sciences agricoles, agroalimentaires et des forêts (DISAFA), université de Turin, 10095 Grugliasco TO, Italie \\ 2 Centre interdisciplinaire de recherche et d'études sur les femmes et le genre (CIRSDe), Centre interdépartemental de recherche et \\ coopération avec les pays du Sahel et de l'Afrique de l'Ouest (CISAO), université de Turin, 10153 Torino TO, Italie \\ 3 Cirad, UMR TETIS, 34398 Montpellier, France \\ ${ }^{4}$ Cirad, UMR Innovation, 34398 Montpellier, France \\ 5 Université de Montpellier, Montpellier, France \\ ${ }^{6}$ Université fédérale du Pará, 66075-110 Belém PA, Brésil
}

\begin{abstract}
Résumé - L'agriculture mondiale est confrontée à de nombreux défis en termes de souveraineté alimentaire. Il s'agit d'abord d'assurer un accès équitable à la nourriture et aux produits agricoles de première nécessité pour une population mondiale croissante. Il s'agit ensuite d'inclure la production agricole dans une perspective plus générale de lutte contre la pauvreté, l'inégalité et la malnutrition dans le monde. Il s'agit enfin de contribuer à une gestion plus durable des ressources naturelles et agricoles et de la biodiversité. L'approche agroécologique semble être l'une des options les plus appropriées pour atteindre ces objectifs, en raison de son caractère holistique concernant l'utilisation des ressources et des processus naturels, qui profite à la fois aux agriculteurs, à l'agriculture durable et à des modèles de consommation alimentaire plus sains. En outre, l'agroécologie est conçue pour soutenir des relations sociales plus égalitaires, en particulier entre hommes et femmes. Néanmoins, l'analyse des relations et des implications entre l'agroécologie et les questions de genre reste une question relativement inexplorée. L'objectif de cet article est donc d'analyser de manière critique et de mettre en évidence les principales opportunités et contraintes de l'agroécologie en termes de durabilité et d'égalité des sexes. La recherche a été réalisée dans le cadre d'expériences agroécologiques menées dans le Nord-Est de l'Amazonie brésilienne, où les agricultrices pratiquent principalement l'extractivisme et où l'agroécologie est encore dans une phase d'émergence comparativement à d'autres régions du pays. Pourtant, malgré leur faible reconnaissance et leur accès limité aux ressources, les femmes contribuent activement à la conservation de la biodiversité et au transfert des connaissances traditionnelles.
\end{abstract}

Mots clés : agroécologie / égalité de genre / Brésil / développement durable

\begin{abstract}
Gender issues and sustainable development: the potential of agroecology in the northeast of Pará, Brazil. World agriculture is facing many challenges in terms of food sovereignty. Firstly, the need to ensure a fair access to food (and good food) and basic necessities to a growing global population. Second, the necessity to include such actions within a more general perspective of dealing with poverty, inequality, and malnutrition worldwide, as well as contributing to a more sustainable management of natural and agricultural resources and biodiversity. The agroecological approach seems one of the most suitable option, because of its holistic nature regarding the use of natural resources and processes, which benefits farmers, sustainable agriculture and healthy food consumption models. In addition, agroecology is designed to support more egalitarian social relations, such as those related to gender equality. Nonetheless, the analysis of the relations and the implications between agroecology and gender issues is still a quite unexplored issue. Therefore, the aim of this article is to critically analyse and highlight the main opportunities and constraints of agroecology in terms of greater sustainability and gender equality as well.
\end{abstract}

\footnotetext{
* Auteur de correspondance : alicentrone@gmail.com

francescaalice.centrone@unito.it
} 


\begin{abstract}
The research was realized within some agroecological experiences carried out in the northeast of Brazilian Amazonia, where women farmers work mostly in the extractive agriculture and where agroecology is still in a preliminary phase by comparison with other regions of the country. Despite their scarce acknowledgment and access to resources, local women actively contribute to the biodiversity conservation and the transfer of traditional knowledge.
\end{abstract}

Keywords: agroecology / gender equality / Brazil / sustainable development

\section{Enjeux et problématique}

Les objectifs du développement durable (ODD) ont été adoptés par l'ONU en septembre 2015. L'objectif $n^{\circ} 5$ fait référence, d'une part, à l'égalité entre les femmes et les hommes et, d'autre part, à l'autonomie des femmes. De fait, ces deux thématiques sont transversales à tous les autres ODD, car la mise en œuvre du développement durable passe nécessairement par elles. L'étude du genre se réfère ici à l'analyse des relations et des différences entre les sexes. Dans l'agriculture, les femmes font face à d'importantes contraintes en termes d'accès, de gestion et de maîtrise réelle des ressources tangibles (terre, crédit, intrants, technologie, etc.) et intangibles (services de vulgarisation, formations techniques, innovation, etc.) (Cornwall, 2016). Malgré une inflexion des programmes de développement rural depuis les années 1990, les femmes disposent de peu de pouvoirs de décision et peinent à faire reconnaître leurs spécificités, par exemple leur plus grande charge de travail (Meinzen-Dick et al., 2014).

L'agroécologie, quant à elle, est souvent présentée comme un moyen pour atteindre les ODD. Ensemble de bonnes pratiques pour le développement durable (FAO, 2011), l'agroécologie favorise des modèles de production et de consommation alimentaire plus sains, plus inclusifs socialement et plus respectueux des ressources naturelles (Altieri et Toledo, 2011). Elle serait particulièrement adaptée aux petits agriculteurs et agricultrices (Lappé, 2016). Dans une vision plus politique, l'agroécologie est aussi un mouvement social pour la promotion de formes plus égalitaires de développement. Dalle et Walsh (2015) ont montré que l'agroécologie peut améliorer les rapports de genre, en permettant aux femmes d'atteindre une meilleure position économique et sociale. L'agroécologie serait un moyen d'éviter les mécanismes d'exclusion technologique explicités ci-dessus. Mais cette amélioration resterait cependant limitée (Nobre, 2005).

Leach (2007) défend l'idée que les programmes de développement promouvant le lien entre femmes et environnement leur ont conféré la responsabilité de «sauver l'environnement» sans leur fournir les ressources nécessaires. Le «succès» de ces programmes a souvent été obtenu aux dépens des femmes, en mobilisant leur travail, non rémunéré. De nouvelles tâches ont été ajoutées à la longue liste de celles «bénévoles» que les femmes assurent. Selon Prévost et al. (2014), dès que les exploitations agroécologiques atteignent de meilleurs niveaux de performance, les hommes redeviennent les protagonistes des prises de décision. L'agroécologie resterait sujette à un monde "masculin» (Rosset et al., 2011), car basé sur la division traditionnelle du travail agricole (Prévost et al., 2014) et sur des dominations ancrées dans le temps. En conséquence, les rapports inégalitaires de genre sont dénoncés par les mouvements militants, en particulier par
l'Association brésilienne d'agroécologie (ABA, 2018), qui qualifie la société de "fermée», traditionnelle et «machiste», avec des cas de violence domestique fréquents.

Au Brésil, l'agroécologie a été reconnue par le gouvernement fédéral, à travers des politiques $a d$ hoc pour assurer son développement. Mais comme ailleurs (Boserup et Kanji, 2007), les femmes ne sont toujours pas complètement acceptées comme de «vrais agriculteurs». Leur contribution reste peu visible dans les statistiques officielles, même si des politiques publiques spécifiques leur sont dévolues. Selon la FAO (2013), $13 \%$ des agriculteurs étaient des agricultrices au Brésil, alors que Butto et Dantas (2011) évaluaient à $50 \%$ le taux des femmes actives dans la production autoconsommée, et à $65 \%$ le taux des femmes travaillant sans rémunération, notamment dans l'horticulture, la sylviculture et l'élevage de petits animaux. Cette invisibilité des femmes agricultrices dans les statistiques agricoles officielles influence les orientations des politiques publiques. Cela est vrai aussi pour l'agroécologie. De fait, la question du lien entre l'agroécologie et l'égalité de genre se pose de plus en plus (Lima et de Jesus, 2017).

Dans cet article, nous faisons l'hypothèse que l'agroécologie constitue un moyen de remédier aux inégalités de genre. Pour vérifier cette hypothèse, deux questions structurent notre réflexion: en quoi les représentations qu'ont les acteurs de terrain du rôle des hommes et des femmes en agriculture influent-elles sur les inégalités de genre? Comment l'agroécologie, et en particulier l'action des mouvements collectifs féminins, remet-elle en question ces inégalités? Notre objectif est de définir à quelles conditions l'agroécologie peut constituer un espace d'opportunité et/ou de contrainte à l'égalité entre femmes et hommes. Le présent travail se veut une contribution originale pour mieux documenter le lien entre agroécologie et genre, encore rare dans la littérature. La structure de l'article reprend les deux questions de recherche formulées, après avoir présenté la méthodologie et le municipe de Santa Luzia, situé en Amazonie brésilienne, où s'est déroulée la recherche.

\section{Cadre d'analyse et méthodologie}

Nos recherches ont été menées en Amazonie, dans le municipe de Santa Luzia do Pará et à Belém, la capitale régionale, dans le cadre du dispositif en partenariat «Amazonie» réunissant l'UFPA (Universidade Federal do Pará), l'EMBRAPA Amazonie orientale (Empresa Brasileira de Pesquisa Agropecuária) de Belém et le Cirad (Centre de coopération internationale en recherche agronomique pour le développement). Les travaux à Santa Luzia avaient pour objectif de caractériser les rapports de genre dans les différents systèmes agricoles et dans les représentations collectives. Les 
activités à Belém consistaient à recueillir les témoignages de représentants des institutions. Le réseau Rede Bragantina de Economia Solidaria (Rede Bragantina) a facilité la logistique et les contacts. Ce réseau regroupe des associations très diverses, dont certains membres font partie de quilombolas (communautés d'Afro-descendants ayant un droit d'accès collectif à la terre, reconnu par la constitution de 1988). Parmi les associations du réseau, certaines sont particulièrement actives: une coopérative de producteurs avec 50 membres actifs (dont dix femmes), une association féminine, une association d'appui en apiculture et production de miel et un centre de formation par alternance (ECRAMA).

Une approche qualitative a été privilégiée, favorisant l'analyse des perceptions diverses des acteurs locaux. Nous avons appliqué une grille d'analyse pluridisciplinaire qui impliquait différentes catégories sociales: des communautés quilombolas, des agricultrices et des agriculteurs familiaux, des représentants des services techniques, des activistes, des experts, des politiciens. Dix-sept entretiens individuels ont été menés, avec des personnes-ressources représentatives de ces différentes catégories, dont $90 \%$ de femmes. Deux questionnaires semi-structurés ont été utilisés, l'un pour les producteurs/productrices et l'autre pour les représentants des institutions. Des récits de vie ont été recueillis auprès des productrices âgées de 65 à 80 ans environ (soit $20 \%$ des personnes interrogées). Le questionnaire était divisé en quatre sections principales : perceptions générales sur l'agroécologie ; développement durable et questions de genre; pratiques agroécologiques et genre; impacts et perspectives selon une approche genre. À Santa Luzia, nous avons aussi animé quatre focus groups : trois avec des producteurs et des productrices en agroécologie, des représentants de coopératives et d'associations, et des chercheurs; un avec des femmes engagées localement dans la politique et le syndicalisme.

\section{Le Nordeste Paraense et la commune de Santa Luzia}

Le territoire du Nordeste Paraense est composé de 20 municipalités avec une population totale de 734545 habitants, dont $48 \%$ vivant dans des zones rurales. Le municipe de Santa Luzia do Pará se situe à environ $200 \mathrm{~km}$ à l'Est de Belém. Il est traversé par la route fédérale BR-316 qui connecte Belém à São Luis. Il s'étend sur une surface de $1356 \mathrm{~km}^{2}$, avec une population de 19348 habitants (en 2016), équitablement partagée entre hommes et femmes et entre ruraux et urbains. La densité est de 14,3 habitants $/ \mathrm{km}^{2}$ (FAPESPA, 2016). La population rurale est passée de $60 \%$ en 2000 à $46 \%$ en 2010 (FAPESPA, 2016). Les activités sont agricoles et d'extraction forestière (bois et produits non ligneux).

Cette zone, colonisée au moment du boom de l'hévéa, est devenue un territoire d'agriculture familiale où coexistent agricultures familiale et capitaliste. L'agriculture familiale présente des statuts divers : quilombos, villages amérindiens, petits villages d'anciens migrants et 13 périmètres de réforme agraire, trois étant gérés par des femmes (données de terrain). Les communautés anciennement installées détiennent une grande connaissance de la nature et de ses ressources, notamment des plantes et produits de la forêt, utilisés à des fins alimentaires, médicales et esthétiques. Selon nous, les systèmes agricoles et extractivistes de l'agriculture familiale relèvent de l'agroécologie, car ils n'utilisent pas ou peu d'intrants chimiques, ils valorisent la biodiversité par des systèmes diversifiés, ils recherchent l'autonomie des exploitations agricoles et la valorisation des produits par des circuits courts et défendent des valeurs de solidarité.

\section{Les représentations locales de l'agroécologie: la femme toujours vouée à l'économie domestique?}

\subsection{Des représentations différentes de l'agroécologie}

Les représentations locales sont très diverses, qu'elles concernent l'agroécologie ou le statut et le rôle des femmes. Pour tous les acteurs interrogés, l'agroécologie se caractérise d'abord par la non-utilisation de pesticides. Mais d'autres caractéristiques sont mises en avant. Certains, notamment les responsables des associations et certains élus, insistent sur le caractère politique de l'agroécologie en tant que mouvement de transformation sociale. D'autres, comme les producteurs du marché biologique de Belém, valorisent l'impact économique. L'agroécologie facilite la vente de produits de qualité en instituant des réseaux de confiance avec les acheteurs. D'autres encore, en premier lieu les femmes productrices, insistent sur les bénéfices en termes de santé, dus à une alimentation saine. Ces différents regards montrent que l'agroécologie est traversée par les tensions et les débats habituels sur les équilibres à trouver entre les différents piliers du développement durable. S'il y a un consensus sur le respect de l'environnement, le pilier économique peut dominer le pilier social ou l'insertion au marché la solidarité.

La dimension politique de l'agroécologie est davantage présente chez les acteurs institutionnels et les responsables des associations - des militants se voulant porteurs d'un projet de société. Les producteurs de base, quant à eux, soulignent surtout les aspects agricoles et environnementaux. La distinction de représentation entre ces types d'acteurs est plus significative que les différences de genre. Femmes et hommes tiennent des discours proches sur l'agroécologie. Ainsi, les présidentes des associations, membres du réseau Bragantina, défendent le caractère politique de l'agroécologie. En revanche, cet aspect est généralement peu affirmé chez les productrices ou les vendeuses du marché de Belém. Avec les hommes, elles se présentent comme de «vrais entrepreneurs et entrepreneuses » agricoles, principalement préoccupés de la durabilité économique de leurs activités. Mettant en avant leurs pratiques agricoles, elles préfèrent d'ailleurs utiliser le terme « agriculture biologique ».

Ces divergences se retrouvent dans les activités menées avec Natura, une entreprise multinationale brésilienne de production de cosmétiques naturels. Les femmes quilombolas de Santa Luzia do Pará collectent les graines de murumuru (Astrocaryum murumuru) pour cette entreprise. Le beurre qui en est extrait est utilisé dans les produits hydratants. L'intérêt de travailler avec une multinationale comme Natura fait débat au sein des communautés. Pour certains, cette coopération pourrait conduire à un abandon des valeurs initiales de l'agroécologie (notamment celles autour de la protection des savoirs locaux) en favorisant un «mauvais » développement. 
Mais ce débat oppose surtout les producteurs et productrices ayant réussi économiquement aux responsables des associations. Les autres restent assez étrangers à ce débat. Le même décalage se retrouve quand les entretiens abordent les thèmes du développement durable. Le discours des responsables des associations reprend les enjeux de l'agenda international. Mais ce discours peine à intégrer des réponses concrètes aux questions sociales, à la pauvreté, à l'accès au marché et aux droits élémentaires, des réalités que vivent les producteurs de base.

\subsection{Femmes et agroécologie: un manque de reconnaissance?}

La très grande majorité des entretiens mettent l'accent sur l'attitude différente des hommes et des femmes face à la nature, au travail et, par conséquent, aux techniques productives. «Les hommes ont une interprétation technique » et très transformatrice «... si quelque chose ne marche pas, il faut changer... tandis que la femme est plus constante, patiente, plus ouverte et flexible aux changements et aux innovations ». Le lien fort entre les femmes et la dimension environnementale de l'agroécologie est aussi mis en avant. Les femmes sont moins mobiles et souffrent d'isolement : «Ici dans l'Amazonie les hommes migrent, se déplacent davantage pour aller cultiver et travailler ailleurs... tandis que les femmes restent à la maison. » Ceci expliquerait que les femmes choisissent des activités davantage en lien avec l'agroécologie (potager, plantes médicinales), qui nécessitent davantage de travail minutieux et de patience.

Les femmes sont reconnues comme premières porteuses de la sauvegarde de la biodiversité. Les hommes ne disposeraient pas de ces connaissances: «Moi, je cultivais une plante sur notre terrain, plante dont ma mamie m'avait parlé, mais mon mari ne la connaissait pas et l'a coupée !» Ces connaissances présentent une forte connotation de genre car elles sont transmises de génération en génération, notamment de mère à fille et de tante à nièce. Toutes les femmes rencontrées, même celles qui ne sont pas directement productrices, peuvent reconnaître, collecter et utiliser un grand nombre de variétés différentes d'arbres, de plantes, d'herbes, de fruits et de semences. Elles ont aussi les compétences nécessaires pour préparer les médicaments traditionnels à base de produits de la forêt.

Les entretiens montrent que la valeur des activités productives des femmes est peu prise en compte. L'«agroécologie des femmes », liée aux jardins familiaux et à la collecte des plantes médicinales, n'est pas reconnue comme une activité motrice de l'économie des ménages. Les femmes sont d'ailleurs souvent les premières à ne pas valoriser leurs contributions à l'économie familiale : «Nous ne donnons pas de valeur au travail de nos femmes... Pour changer [...], nous devons partir des comportements machistes présents dans nos familles!» $\mathrm{Ce}$ manque de reconnaissance touche aussi les rôles politiques et économiques des femmes. Les femmes sont très peu présentes dans les instances de décision. Elles ne sont que $20 \%$ dans les exécutifs et les législatifs municipaux. Dans ces exécutifs, elles ne sont que très rarement en charge des secteurs clés comme l'économie. Elles sont peu représentées dans les directions des instituts techniques ou dans les associations, sauf celles uniquement féminines. Le manque de reconnaissance concerne aussi la gestion économique des exploitations. Ainsi, malgré son succès économique d'entrepreneuse, une veuve expose ses difficultés à être considérée comme la chef de l'exploitation aux yeux de ses ouvriers, qui ont du mal à accepter ses ordres.

Cette représentation mitigée du rôle de la femme dans l'agroécologie perpétue les fortes contraintes en termes de production. Quatre inégalités sont habituellement identifiées par la littérature (Milgroom, 2015): l'accès à la terre, aux intrants, au crédit, à l'éducation et aux services de vulgarisation. L'accès à la terre est peu contraignant dans les quilombolas où les terres sont collectives. Les trois autres contraintes constituent des obstacles pour l'ensemble des producteurs locaux (femmes et hommes inclus). À Santa Luzia, la principale contrainte citée dans les entretiens est celle liée aux services de vulgarisation et d'appui spécifique aux femmes. La proportion de femmes employées dans les agences d'assistance technique (EMATER) est de $30 \%$ au niveau national. Elles sont surtout en charge du domaine social (Ferro, 2014). Les femmes agricultrices ne sont que très rarement les bénéficiaires de formations spécifiques agricoles. Les vulgarisateurs, selon les femmes interrogées, suggèrent de façon plus ou moins implicite aux femmes d'effectuer des choix productifs et des demandes de crédit «complémentaires à ce que font leurs maris!», notamment au sein du PRONAF (Programa Nacional de Fortalecimento da Agricultura Familiar)-Mulher (une mesure de crédit pensée expressément pour les femmes). Or, selon Rieu et Dahache (2008), la notion de complémentarité cache souvent le refus de l'égalité. Ainsi, la femme mariée ne peut toujours pas demander un crédit PRONAF sans se présenter avec son mari.

En revanche, il n'y a pas de discrimination entre hommes et femmes pour les droits de succession sur la terre. Les différenciations sociales sont ici plus importantes que les inégalités entre les genres. De nombreuses femmes sont «propriétaires» de la terre héritée de leurs parents. Mais, comme les hommes, elles ne disposent pas toujours des titres de propriété leur permettant d'avoir accès plus facilement aux financements. Il est vrai qu'en Amazonie, les organisations traditionnelles sont basées sur des répartitions relativement égalitaires des rôles dans l'agriculture. En particulier, les communautés quilombolas donnent plus d'espace et de pouvoir aux femmes que l'agriculture familiale.

\section{Les actions du mouvement agroécologique sont-elles efficaces pour lever ces contraintes?}

La participation des agricultrices aux luttes sociales rurales et aux expériences du mouvement agroécologique est très ancienne au Brésil. Cependant, le rôle des femmes rurales au sein des mouvements sociaux n'a été reconnu formellement qu'à partir des années 1980 (Siliprandi, 2015). L'organisation des mouvements féminins ruraux s'est structurée au cours de la décennie 1990. En tout état de cause, en Amazonie et dans le Pará, plusieurs réseaux, souvent exclusivement féminins, ont été créés dans les années 1990 (García Roces et al., 2014). Les femmes occupent des positions de premier plan dans le MMNEPA (Mouvement des femmes du Nord-Est du Pará) ou la Rede Bragantina. Le but principal de ces réseaux est de 
favoriser les échanges de connaissances et le renforcement de compétences, principalement autour des produits et pratiques agricoles. Mais d'autres thèmes sont abordés comme la certification sociale participative. Les enquêtes permettent de juger de l'impact de ces actions.

En premier lieu, plus que les hommes, les femmes manifestent un intérêt pour les questions environnementales et la qualité des aliments. Mais cela ne signifie pas qu'elles choisiraient toujours les pratiques agroécologiques même si elles avaient un pouvoir et un accès aux ressources tangibles (technologies, machines...) identiques aux hommes.

En deuxième lieu, les personnes interrogées ont souligné le lien entre, d'une part, pratiques agroécologiques et autonomie, et, d'autre part, entre pratiques agroécologiques et poids des femmes dans la société. C'est d'abord le gain de liberté individuelle obtenu grâce aux activités d'agroécologie qui est souligné. Les femmes peuvent compter sur des revenus personnels. Dans les communautés quilombolas, toutes les femmes ont déclaré pouvoir décider de façon autonome la destination des revenus provenant de cette activité : «mon travail, mon argent ».

Un troisième élément est l'impact des formations sur les pratiques agricoles et surtout sur la transformation des produits, activité de valorisation encouragée dans le réseau Bragantina et dans laquelle les femmes ont un rôle majeur. Les thématiques de contrôle de la qualité et de la sécurité alimentaire des produits sont prépondérantes: "Avant, j'utilisais n'importe quel type de bouteille pour garder l'huile de coco ; maintenant, après la formation, j'utilise des bouteilles foncées. » Les réseaux contribuent aussi à la construction de relations de confiance par la connaissance personnelle entre vendeur et acheteur. La mise en réseau renforce un sentiment d'appartenance commune, en donnant plus de valeur (même symbolique) et de conscientisation aux femmes impliquées. L'artisanat, la sécurité au travail, la gestion environnementale, la commercialisation de produits et l'utilisation des technologies de l'information et de la communication (TIC) sont aussi des thèmes de formation: « Je cultive comme ma grandmère, mais j'utilise WhatsApp pour recevoir les commandes. » Les formations permettent également d'aborder les droits individuels et la violence domestique. Elles s'adressent aussi aux jeunes, afin de limiter leur exode rural. C'est notamment l'objectif de l'école ECRAMA, où les élèves sont des jeunes de moins de 25 ans, avec une légère prédominance de garçons. C'est également l'objectif des écoles des quilombolas, où l'institutrice est généralement une jeune femme issue de la communauté.

Un autre point important de la situation des femmes est la violence domestique, souvent liée à l'alcoolisme. Faire partie de réseaux agroécologiques permet aux femmes d'accéder à des formes de protection, de partage et de conscientisation collective. Mais en cas d'augmentation de cette violence, qui peut d'ailleurs résulter directement de la participation aux activités des associations, le manque de mesures spécifiques, d'accompagnement et de soutien aux femmes battues est patent.

\section{Discussion et conclusions}

L'agroécologie développée dans le municipe de Santa Luzia contribue au renforcement de l'indépendance écono- mique des femmes et de leur rôle dans les prises de décisions, tant dans la famille que dans les instances collectives (associations, réseaux). L'agroécologie mise en avant est une agroécologie d'extractivisme, où les femmes occupent un rôle historiquement déterminant dans des rapports entre hommes et femmes plus égalitaires qu'ailleurs. Le renforcement des compétences, axe privilégié du mouvement et des réseaux agroécologiques, a favorisé la prise de conscience des femmes, leur autonomie financière, leur accès au marché, leur prise de responsabilité dans les instances de gestion et, en conséquence, un pouvoir de décision renforcé dans les activités agricoles.

Comment renforcer ces évolutions positives? Faut-il prioritairement consolider les pratiques égalitaires existantes dans les activités extractivistes en les étendant aux autres activités? Ou faut-il engager une réflexion globale pour modifier les pratiques de gestion agricoles de l'exploitation? Il serait alors nécessaire d'insérer l'égalité de genre et la valorisation du travail des femmes comme critères de certification et donc de qualification des produits dans les mouvements agroécologiques. C'est le mot d'ordre que revendiquent les femmes du MST (Mouvement des sans terre): «Pas d'agroécologie sans féminisme» (Prévost et al., 2014.). Ces critères viendraient s'ajouter aux critères techniques, notamment de non-utilisation de pesticides. Cela implique aussi des conditions égales de revenus et de droits entre hommes et femmes, une participation réelle des femmes au sein des institutions d'intermédiation agricoles (coopératives de commercialisation, services de vulgarisation, etc.), et une lutte contre toutes les formes de violence contre les femmes.

L'analyse de l'impact des expériences agroécologiques en termes d'égalité de genre conduit à un certain nombre de constatations :

- l'insuffisante «quantification» de la présence des femmes en agroécologie, en particulier dans les statistiques officielles, où le manque de données désagrégées par sexe traduit le manque de valorisation et de reconnaissance du rôle des femmes dans l'agroécologie;

- dans cette perspective, il est nécessaire de proposer et d'aboutir à des outils de suivi-évaluation et d'accompagnement autour du genre et de l'agroécologie, outils qui puissent aussi favoriser et accompagner l'institutionnalisation de l'agroécologie;

- la richesse amenée par les perceptions et perspectives des acteurs locaux doit être prise en considération dans le processus d'élaboration des politiques et des outils de suivi-évaluation.

Dans cette perspective, la proposition d'un cadre d'analyse de genre - y compris des indicateurs - à appliquer spécifiquement aux expériences agroécologiques serait à développer dans les prochaines étapes de la recherche. Un autre chantier est celui de la pérennité et de la continuité des actions. Cela pose la question de la transmission des savoirs et des connaissances vers les jeunes, mais aussi des jeunes élèves vers les adultes et leurs familles ou communautés d'origine. Cette interaction entre les différentes classes d'âge est porteuse de reconnaissance des genres et d'une meilleure « égalité entre femmes et hommes, mais aussi entre adultes et jeunes ». Elle est aussi porteuse d'améliorations des pratiques environne- 
mentales. Le vrai défi est donc de donner plus de voix et de poids aux jeunes et aux femmes, en tant que porteurs de changement, agissant par là comme un pont entre tradition et innovations durables.

\section{Références}

Altieri MA, Toledo VM. 2011. The agroecological revolution in Latin America: rescuing nature, ensuring food sovereignty and empowering peasants. Journal of Peasant Studies 38(3): 587-612. DOI: 10.1080/03066150.2011.582947.

Associação Brasileira de Agroecologia (ABA). 2018. Como pode se fazer agroecologia sem as mãos e os olhares das mulheres? http:// aba-agroecologia.org.br/wordpress/como-pode-se-fazer-agroecolo gia-sem-as-maos-e-os-olhares-das-mulheres/ [2018/06/11].

Boserup E, Kanji N. 2007. Woman's role in economic development. London, UK: Earthscan, $270 \mathrm{p}$.

Cornwall A. 2016. Women's empowerment: what works? Journal of International Development 28(3): 342-359. DOI: 10.1002/ jid.3210.

Butto A, Dantas I. 2011. Autonomia e cidadania políticas de organizaço produtiva para as mulheres no meio rural. Brasília, DF (Brasil): Ministério do Desenvolvimento Agrário (Brasil) IICA.

Dalle SP, Walsh S. 2015. USC Canada's experience in supporting community seed banks in Africa, Asia and the Americas. In: Vernooy R, Shrestha P, Sthapit B, eds. Community seed banks. Origins, evolution and prospects. New York, USA: Routledge.

Fundação Amazônia de Amparo a Estudos e Pesquisas (FAPESPA). 2016. Estatistica municipal. Santa Luzia do Pará, Bélem: FAPESPA.

Food and Agricultural Organisation (FAO). 2011. The State of food and agriculture: women in agriculture. Closing the gender gap for development. Rome, Italy: FAO.

Food and Agricultural Organisation (FAO). 2013. No Brasil, 13\% dos agricultores são mulheres. Notícias FAO. http:/www.fao.org/ americas/noticias/ver/es/c/230178/ [2017/10/18].

Ferro S. 2014. Estudio comparativo regional de asistencia técnica y extensión rural con perspectiva de género. Programa Regional de Género de la Reunión Especializada de la Agricultura Familiar del
Mercosur (REAF). https://dspace.unila.edu.br/xmlui/bitstream/ handle/123456789/1742/FERRO\%20Asistencia\%20Tecnica\%20y $\% 20$ Extensi $\%$ C3\%B2n\%20Rural\%20estudio\%20comparativo \% 20regional.pdf?sequence=1 [2017/09/23].

García Roces I, Soler Montiel M, Sabuco i Cantó A. 2014. Perspectiva ecofeminista de la Soberanía Alimentaria: la Red de Agroecología en la Comunidad Moreno Maia en la Amazonía brasileña. Relaciones Internacionales 27: 75-96.

Lappé FM. 2016. Farming for a small planet: agroecology now. Development 59(3-4): 299-307.

Leach M. 2007. Earth mother myths and other ecofeminist fables: how a strategic notion rose and fell. Development and Change 38(1): 67-85. DOI: 10.1111/j.1467-7660.2007.00403.x.

Lima MMT, de Jesus VB. 2017. Questões sobre gênero e tecnologia na construção da agroecologia. Scientiae Studia 15(1): 73-96. DOI: 10.11606/51678-31662017000100005.

Meinzen-Dick R, Kovarik C, Quisumbing AR. 2014. Gender and sustainability. Annual Review of Environment and Resources 39(1):29. DOI: https://doi.org/10.1146/annurev-environ101813-013240.

Milgroom J. 2015. Women forging change with agroecology. Farming Matters 31(4): 1-48.

Nobre M. 2005. Quand la libération des femmes rencontre la libération des semences. Mouvements 4: 70-75. DOI: 10.3917/ mouv.041.0070.

Prévost H, Esmeraldo GGSL, Guetat-Bernard H. 2014. Il n'y aura pas d'agroécologie sans féminisme: l'expérience brésilienne. Pour 2 (222): 275-284. DOI: 10.3917/pour.222.0275.

Rieu A, Dahache S. 2008. S'installer comme agricultrice: sur la socialisation et la formation sexuée en agriculture. Revue d'études en agriculture et environnement 88(3): 71-94.

Rosset PM, Machin Sosa B, Roque Jaime AM, Ávila Lozano DR. 2011. The Campesino-to-Campesino agroecology movement of ANAP in Cuba: social process methodology in the construction of sustainable peasant agriculture and food sovereignty. The Journal of Peasant Studies 38(1): 161-191. DOI: 10.1080/ 03066150.2010 .538584 .

Siliprandi E. 2015. Mulheres e agroecologia: transformando o campo, as florestas e as pessoas. Brazil: Editora UFRJ, 356 p.

Citation de l'article : Centrone FA, Tonneau J-P, Piraux M, Cialdella N, De Sousa Leite T, Mosso A, Calvo A. 2018. Questions de genre et développement durable: le potentiel de l'agroécologie dans le Nordeste du Pará, Brésil. Cah. Agric. $27: 55003$. 